ORIGINAL RESEARCH ARTICLE

\title{
Integrating Family Planning and HIV Services at the Community Level: Formative Assessment with Village Health Teams in Uganda
}

\author{
Aurélie Brunie ${ }^{1}$, Patricia N. W. Mucheri ${ }^{2}$, Angela Akol ${ }^{3}$, Mario Chen ${ }^{4}$, Sarah J. Mercer ${ }^{5}$, and \\ Tricia Petruney ${ }^{6}$
}

Health Services Research, FHI $360^{1}$; Formerly with FHI 360 Uganda; now with Clinton Health Access Initiative ${ }^{2}$; FHI 360, Uganda 3 ; Biostatistics, FHI $360^{4}$; Formerly with FHI $360^{5}$; Research Utilization, FHI $360^{6}$

*For Correspondence: Email: abrunie@fhi360.org; Phone: +1 2028848982

\begin{abstract}
Little is known on integrating HIV and family planning (FP) services in community settings. Using a cluster randomized controlled design, we conducted a formative assessment in two districts in Uganda where community health workers, called VHTs, already offered FP. Thirty-six trained VHTs also provided HIV testing and counseling (HTC) during the intervention. We surveyed all 36 VHTs and 256 FP clients, and reviewed service statistics. In the intervention group, VHTs tested $80 \%$ of surveyed clients for HIV, including $76 \%$ they already saw for FP and 22\% who first came to them for HTC before receiving FP. Comparing clients' experiences in the intervention and control groups, adding HTC does not appear to have negatively affected FP service quality. VHTs reported more monthly clients, but rated their workload as easy to manage. This integrated model seems feasible and beneficial for both VHTs and clients, while not resulting in any negative effects. This study was registered with ClinicalTrials.gov, number [NCT02244398]. (Afr J Reprod Health 2017; 21[2]: 73-80).
\end{abstract}

Keywords: family planning, HIV, community health workers, integration, Uganda

\section{Résumé}

Peu d'informations sont disponibles sur l'intégration des services de planification familiale (PF) et du VIH dans les milieux communautaires. En utilisant un plan randomisé contrôlé par grappes, nous avons mené une évaluation formative dans deux districts en Ouganda dans lesquels les agents de santé communautaire qui sont appelés VHT offraient déjà des services de PF. Trente-six VHT formés ont également fourni des services de conseil et dépistage du VIH (CDV). Nous avons enquêté les 36 ASC et 256 clientes PF, et nous avons examiné les statistiques sur les services. Dans le groupe d'intervention, les VHT ont testé $80 \%$ des clients enquêtées pour le VIH, y compris $76 \%$ que les VHT voyaient déjà pour la PF et $22 \%$ qui sont d'abord venus pour le CDV avant avant de recevoir la PF. En comparant les expériences des clientes dans les groupes avec et sans intervention, l'ajout du CDV ne semble pas avoir affecté négativement la qualité des services PF. Les VHT ont signalé plus de clients mensuellement, mais ont évalué leur charge de travail comme étant aussi facile à gérer. Ce modèle intégré semble faisable et bénéfique pour les VHT et les clients, et sans entraîner d'effet négatif. Cette étude a été enregistrée auprès de ClinicalTrials.gov, numéro [NCT02244398]. (Afr J Reprod Health 2017; 2112]: 73-80).

Mots clés: planification familiale, VIH, agents de santé communautaires, intégration, Ouganda

\section{Introduction}

Policy and financial support for the integration of family planning (FP) and HIV services has grown substantially in recent years ${ }^{1,2}$. Research and programmatic experience have shown that integrated FP/HIV service delivery is a worthwhile strategy to comprehensively address clients' sexual and reproductive health needs ${ }^{3-5}$. This is especially the case in settings with high unmet need for FP and high HIV prevalence, like sub-
Saharan Africa ${ }^{6}$. Although conclusions are complicated by the heterogeneity of interventions, settings, and studies, research has demonstrated that linkages between FP and HIV services increased uptake of services and held promise for promoting dual method use ${ }^{3-5}$.

Completed research on integrated HIVreproductive health services has been conducted almost exclusively in health facilities ${ }^{3,4}$. Largely missing from the evidence base on integration are cases of community-based service delivery. 


\section{Brunie et al}

Community health worker (CHW) programs are a long-standing approach for reaching underserved populations and represent a promising platform for the delivery of integrated FP/HIV services. In light of the health workforce crisis, CHWs' responsibilities are being reconfigured toward an increasingly substantial role in service delivery in several settings ${ }^{7,8}$. Despite calls for task-shifting in both FP and HIV ${ }^{6,9}$, however, the scope of their involvement in integrated approaches has expanded slowly. Consequently, few studies have examined integrated service provision by CHWs. Moreover, studies on CHWs typically examine partial integration models through expansion of existing FP services to include referrals for HIV testing and counseling and supportive care, as opposed to full integration with co-provision of services ${ }^{10,11}$.

This study is a formative assessment of a community-based model of integrated FP/HIV testing and counseling (HTC) service delivery in Uganda. In Uganda, modern contraceptive prevalence is $26 \%$ and HIV prevalence $7.3 \%{ }^{12,13}$. Although more than $90 \%$ of adults know where they can get an HIV test, only $65.8 \%$ of women and $44.9 \%$ of men have ever been tested, and the majority of those tested received only one or two tests in their lifetime ${ }^{13,14}$. The government is rolling out a nationwide Village Health Team strategy, whereby volunteer CHWs, called VHTs, are the primary mechanism for community-based health programming. Some VHTs have been trained in FP and offer those services. This intervention integrated HTC into the FP services offered by VHTs, aiming to increase the range of services available to FP clients, and to reduce barriers to HTC that may stem from poor access or stigma. Integration models span various possible combinations between FP and a range of HIV services, and several delivery strategies, including co-location, co-provision, or referrals. Thus, 'smart' integration is tailored for context, and beneficial and feasible for both clients and providers ${ }^{15}$. This article examines whether community-based FP/HTC is a smart integration model. We report on how integrated service delivery affects opportunities for recruiting clients for both FP and HTC services and, in turn, service uptake. We also report findings on how integration
Community-based FP/HTC provision in Uganda

influences VHTs' and clients' perspectives on service quality and convenience. We further consider synergies in the delivery of integrated services (i.e. the extent to which services delivered were combined and VHTs' knowledge of integrated FP/HIV messages), the impact of the new service on FP service delivery and VHTs' workloads, and the functioning of system factors.

\section{Methods}

This descriptive assessment of community-based integrated FP/HTC as a smart integration model was embedded in a study of the feasibility, acceptability, and effects of HTC service provision by VHTs; those findings are reported elsewhere ${ }^{16}$. The study design was two-arm cluster randomized controlled. VHTs are linked to sub-county level health centers (HC III) for supervision, referral management, and commodity supply for FP. Eight $\mathrm{HC}$ III in two districts were pair-matched (district; timing of introduction of injectable contraceptive service delivery by VHTs; staff HTC qualifications) by the investigators; then HC III in each pair were randomly allocated to intervention or control group using a random number generator. The study included VHTs attached to the selected HC III who had at least six months experience providing FP, including injectable contraceptives. The rationale was to build on selection criteria (e.g. literacy) and strengthened supportive mechanisms for injectable service delivery. In the intervention group, 38 VHTs received classroom training and supervised practical experience in HTC, including both counseling and rapid testing following the national serial algorithm, and integrated FP/HIV messages; 36 VHTs then delivered integrated FP/HTC services, while two women with poor eyesight who had trouble interpreting results were not retained. In the control group, 38 VHTs offered FP only. The number of VHTs balances a desire to produce programmatically meaningful evidence with practical considerations. HTC services were available to all adults in the community, regardless of FP use. System support came from the expansion of existing mechanisms for FP. Specifically, VHTs traveled to health centers monthly to receive support from the midwife on 


\section{Brunie et al}

FP and HIV counseling, and the lab technician on testing aspects; at that time, they also brought waste, submitted their reports, and obtained FP and HTC supplies. The intervention period ran from May 2012 until September 2013, after which all VHTs returned to providing FP only.

Data were collected after about ten months to capture full implementation. Between April and June 2013, trained data collectors conducted structured surveys with all VHTs in the intervention group and with adult, female, revisit FP clients in the intervention and control groups. VHTs in the control group were not interviewed. The primary outcome for the client survey was an HIV testing attitude scale supporting the assessment of the effects of the HTC component on testing attitudes and behaviors (results not reported in this paper): we estimated that completing 30 surveys per HC III would permit detecting a meaningful effect size $(0.5$ standard deviation units between the two groups), with $80 \%$ power, 5\% significance level, and 0.03 intraclass correlation. VHTs in both groups notified their clients of the study in advance and inquired about willingness to be contacted; field supervisors then created a list using VHT records to determine eligibility and randomly selected clients. Interviews took place at the health center (VHTs) or in clients' homes in one of three local languages; all participants received a small cash compensation, as per local ethics guidelines. VHT service statistics were routinely collected and extracted for January through March 2013 for analysis. Makerere University's School of Medicine Research and Ethics Committee, the Uganda National Council for Science and Technology, and FHI 360's Protection of Human Subjects Committee approved this study.

Data were analyzed using SAS 9.3 (SAS Institute, Cary, USA). All analyses presented in this article are exploratory descriptive analyses that we planned ex-ante. First, we produced descriptive statistics for VHTs and clients in the intervention group. Second, we compared clients' experiences receiving FP services between groups using linear mixed models with random effects to adjust for clustering at the VHT and HC III levels to assess possible changes in service quality. Third, we analyzed VHT record data in the intervention
Community-based FP/HTC provision in Uganda

group to describe the distribution of services provided. We considered that clients received FP services if they left with a modern method, and that they received HTC if they were tested by the VHT. This was because of concerns about inconsistent reporting of FP counseling, and to establish some comparability between the two services (i.e. full service provision). Service statistics are reported as number of visits, and not clients, for January to March 2013. Quarterly reporting is programmatically meaningful and may reasonably approximate the number of clients due to the periodicity of injectable contraceptives (the most popular method) and the fact that VHTs encouraged HIV-negative clients to retest after three months. We chose the period to be one with no national stock-outs of either FP or HTC commodities.

\section{Results}

All 36 VHTs and 137 of their family planning clients were surveyed in the intervention group; there were 119 client surveys in the control group. VHTs were predominantly women $(80.6 \%)$; all had attended primary school and $72.2 \%$ secondary school. They were 43.7 years old on average and had been offering injectable contraceptives for 4.6 years.

\section{Opportunities for FP and HTC service uptake}

Of the 36 VHTs in the intervention group, $72.2 \%$ said that most of the women they had tested for HIV were already seeing them for FP. However, most VHTs found it easy to recruit both women who were already their FP clients $(91.6 \%)$ and women who were not for an HIV test $(94.4 \%)$. When asked how adding HTC had affected their ability to recruit FP clients, $91.7 \%$ of VHTs felt it had become easier, and the others reported no change. Among those who felt that recruitment had become easier, the main reasons were having the opportunity to talk about FP to clients coming for an HIV test (69.4\%) and greater exposure in the community (63.9\%). Almost all VHTs (88.9\%) had ever tested someone who subsequently became a FP client, while $13.9 \%$ had lost a FP client after testing her and finding that she was HIV-positive. 


\section{Brunie et al}

Client interviews paint a similar picture. In the intervention group, 110 of $137 \mathrm{FP}$ clients $(80.3 \%)$ had been tested for HIV by the VHT. Among them, $76.4 \%$ were already seeing the VHT for FP when they were tested, $21.8 \%$ came for a test before becoming FP clients, and $1.8 \%$ started receiving both services simultaneously.

\section{Perspectives on service quality and convenience}

According to VHTs, the main advantages to integrated FP/HTC services were convenience (less time seeking services/fewer visits) (88.9\%), better service/advice (69.4\%), and more privacy through reduced need for clients to disclose status to multiple providers $(30.6 \%)$. Other benefits included improved recruitment/confidence by clients (16.7\%) and reduced stigma (16.7\%). The main disadvantages were lack of time (33.3\%) and clients' fear of stigma when seen with the VHT $(16.7 \%) ; 17.1 \%$ of VHTs felt there were no challenges.

FP clients in the intervention group shared similar perspectives. Advantages included less visits/time $(84.7 \%)$, better services/advice $(66.4 \%)$, and reduced stigma (21.2\%). Most $(83.2 \%)$ saw no disadvantages; $5.1 \%$ mentioned concerns about confidentiality and $3.7 \%$ about the VHT being busy. Responses were similar regardless of whether clients had been tested for HIV by the VHT or not.

\section{Synergies in delivering integrated services}

\section{Combination of services}

Table 1 shows numbers of FP and HTC visits over three months; $82.6 \%$ of all visits were with women. Among men, the most common service was HTC only (64.7\% of visits with men); for women it was FP only $(54.1 \%$ of visits with women). Overall, VHTs provided FP only in $47.3 \%$ of all visits, HTC only in $25.1 \%$ of visits, and both services in $22.6 \%$ of visits. An HIV test was received in $32.4 \%$ of the visits during which a FP method was provided; VHTs gave a FP method in $47.4 \%$ of visits during which they tested a client for HIV.
Community-based FP/HTC provision in Uganda

Table 1: Distribution of Client Visits between January and March 2013 in the Intervention Group, by Type of Services Provided.

\begin{tabular}{llllll}
\hline & $\begin{array}{l}\text { FP } \\
\text { only }\end{array}$ & $\begin{array}{l}\text { HTC } \\
\text { only }\end{array}$ & $\begin{array}{l}\text { FP and } \\
\text { HTC }\end{array}$ & Neither & Total \\
\hline Male & 18 & 77 & 17 & 7 & 119 \\
Female & 306 & 95 & 138 & 27 & 566 \\
\hline Total & 324 & 172 & 155 & 34 & 685 \\
\hline
\end{tabular}

${ }^{\text {a }}$ Includes clients that received HTC counseling but were not tested, clients who were not counseled for HTC, and clients with undocumented HTC services. All received a FP method

\section{Delivery of integrated messages}

The VHT survey examined knowledge of integrated messages like FP for people living with HIV and dual protection. While $72.2 \%$ of VHTs correctly said that HIV-positive women could safely use all FP methods, in another question $55.6 \%$ incorrectly agreed with the statement that condoms were the only FP method that HIVpositive women should use. Only $16.7 \%$ knew that it was possible for an HIV-positive woman to give birth to an HIV-negative baby without medical assistance.

When asked about methods offering dual protection against unintended pregnancy and HIV, $72.2 \%$ of VHTs mentioned condoms and $27.8 \%$ condoms along with another FP method (i.e. dual method use). This included eight VHTs (22.2\%) who mentioned both correct responses. Clients' responses support this pattern: while $90.9 \%$ of the clients who had been tested for HIV by the VHT confirmed being counseled on dual protection, when asked about actual methods protecting against both pregnancy and HIV, $72.3 \%$ responded condoms and $22.6 \%$ responded dual method use; $4.4 \%$ of the sample failed to mention either. Seventeen women $(12.4 \%$ of the total sample) gave both correct responses.

\section{Impact on FP service delivery and VHTs' workloads}

Table 2 compares FP service quality between groups through clients' experiences. Nearly all clients were still using a modern method at the time of the survey, with the vast majority reporting injectable use. There were no significant 
Table 2: Client Profile, FP use, and Satisfaction with Services Received, by Group

\begin{tabular}{|c|c|c|c|}
\hline & $\begin{array}{ll}\begin{array}{l}\text { Control } \\
(\mathbf{n}=119)\end{array} & \text { group } \\
\end{array}$ & $\begin{array}{l}\text { Intervention group } \\
(\mathrm{n}=137)\end{array}$ & p-value \\
\hline \multicolumn{4}{|l|}{ Profile } \\
\hline Age, years, mean (SD) & $30.73(1.14)$ & $31.02(0.40)$ & $<.0001$ \\
\hline Number of living children, mean (SD) & $4.26(0.17)$ & $4.98(0.74)$ & \\
\hline Months seeing the VHT for FP, mean (SD) & $2.83(0.04)$ & $3.96(1.15)$ & \\
\hline \multicolumn{4}{|l|}{ Highest level of school attended, $\%$} \\
\hline No schooling & 9.24 & 10.22 & $<.0001$ \\
\hline Primary & 73.95 & 72.26 & 0.0005 \\
\hline Secondary or higher & 16.81 & 17.52 & 0.9715 \\
\hline \multicolumn{4}{|l|}{ FP use } \\
\hline Currently using a modern FP method, \% & 98.3 & 98.5 & 0.8945 \\
\hline FP method(s) used in the past 30 days, $\%^{\mathrm{a}}$ & & & $\mathrm{n} / \mathrm{a}$ \\
\hline Injectables & 95.7 & 94.1 & \\
\hline Oral pills & 3.4 & 4.4 & \\
\hline Male condoms & 5.1 & 3.7 & \\
\hline Implants & 1.7 & 0.7 & \\
\hline \multicolumn{4}{|l|}{ Access } \\
\hline Location of last FP visit with VHT, \% & & & 0.9593 \\
\hline Went to VHT & 93.3 & 93.4 & \\
\hline VHT came to client & 6.73 & 6.6 & \\
\hline $\begin{array}{l}\text { Unable to get method because VHT not available at least } \\
\text { once in past six months, } \%\end{array}$ & 2.5 & 4.4 & 0.1690 \\
\hline $\begin{array}{l}\text { Unable to get method because VHT was out of method at } \\
\text { least once in past six months, } \%\end{array}$ & 23.5 & 16.8 & 0.5268 \\
\hline \multicolumn{4}{|l|}{ Satisfaction } \\
\hline $\begin{array}{l}\text { Satisfied with time VHT spent talking/answering questions, } \\
\%\end{array}$ & 97.5 & 100 & $\mathrm{n} / \mathrm{a}$ \\
\hline Satisfied with FP services received overall, $\%$ & 98.3 & 99.3 & 0.3966 \\
\hline Change in satisfaction with FP services received, $\%^{\mathrm{b}}$ & & & 0.8482 \\
\hline More satisfied now than a year ago & 54.0 & 55.3 & \\
\hline Equally as satisfied & 42.0 & 42.7 & \\
\hline Less satisfied now than a year ago & 4.0 & 1.9 & \\
\hline
\end{tabular}

${ }^{a}$ Of those who were using a FP method at the time of the survey. Multiple responses were possible.

${ }^{\mathrm{b}}$ Of those who had been seeing the VHT for FP for at least a year $(\mathrm{n}=100$ in the control group, $\mathrm{n}=103$ in the intervention group)

${ }^{\mathrm{c}}$ Linear mixed models adjusting for clustering. No other covariates were included.

differences between groups in terms of ability to get a method from the VHT, or of satisfaction. Few FP clients who had been seeing the VHT for over a year, before HTC was added in the intervention group, felt less satisfied compared to a year ago.

Most VHTs described providing both FP (94.4\%) and HTC (88.9\%) services primarily in their own homes. While $83.3 \%$ reported more monthly clients compared to when they were only providing FP, 91.7\% rated their workload as easy to manage. However, two-thirds said it had become more difficult to fulfill their personal responsibilities; one-third said it was equally as difficult as before. All VHTs wished to continue providing HTC. When asked how the added responsibility had affected the quality of their work, $91.7 \%$ said that it had improved, $5.6 \%$ that it was the same, and one VHT that it was not as good as before. All but one felt more appreciated by the community than when they were only offering FP, and $91.7 \%$ reported increased work satisfaction.

\section{Functioning of system factors}

All but two VHTs confirmed reporting to the same health center for both FP and HTC. While 41.7\% verified receiving support from health center staff on counseling and technical aspects of HTC in the six months preceding the survey, $22.2 \%$ said they had received support for only one of those two aspects and $36.1 \%$ for neither. As a reference, 


\section{Brunie et al}

$75.0 \%$ of VHTs reported receiving support from health center staff for FP. Of those, $70.4 \%$ indicated that this support had increased since they also started providing HTC, while $22.2 \%$ said it was the same, and $7.4 \%$ that it had decreased.

Over the six months preceding the survey, VHTs reported being unable to obtain HTC supplies on average 1.6 times when they went to the health center, and 2.3 times for FP commodities. Since the beginning of HTC service provision, half of VHTs had been unable to test a client at least once due to not having all three test kits; half of VHTs had run out of condoms.

\section{Discussion}

Service integration can take multiple forms, and approaches need to be tailored to the local context and available resources and capacity. Our assessment provides rare evidence on a full integration model at the community level. While much of the available literature emphasizes integration of FP into HIV services in facility settings, our model in Uganda used FP as the entry point for integrated service delivery at the community level. Our assessment identified several benefits to this model. Uptake of HTC was high among FP clients, and HTC services also appealed to men. Although the main group reached comprised women already seeing VHTs for FP, we found evidence of bi-directional integration, with the new service contributing to expanding VHTs' FP clientele.

With careful selection and appropriate training and support, task sharing between health center staff and volunteers who are well-connected with their communities is intuitively appealing. Yet as CHWs' role expands in Uganda and other settings, the question of how many tasks they can reasonably and safely carry out simultaneously becomes critical. Continued ability to meet quality standards for previous services and workloads are important considerations. Comparative analyses did not show significant differences in clients' experiences with FP services. Despite an increased monthly client volume, VHTs did not report feeling overwhelmed. This may be partly explained by the minimal transport implications of the new service, both in relation to trips to the

\section{Community-based FP/HTC provision in Uganda}

health center and to movements around the community. Given that transport was the main challenge reported by VHTs in a recent study, this is important ${ }^{17}$. Moreover, VHTs' expanded role appeared to improve their motivation through enhanced visibility and technical skills. This is consistent with other studies showing that status and skills are important motivators for volunteers ${ }^{17-20}$.

The main areas identified for improvement are integrated messages and system support. Simultaneous use of condoms along with an effective FP method is recommended for reducing the risk of unplanned pregnancy and HIV transmission. Yet counseling over-emphasized condoms as a primary FP method at the expense of dual method use. Moreover, for messages to be delivered in an integrated manner (versus only within provision of one or the other service), further emphasis on linkages between FP and HIV may be required during training.

Continued safe and effective communitybased FP/HTC service provision requires suitable supportive mechanisms. Health centers' oversight of VHTs' FP work did not appear to be compromised after HTC was introduced; in fact, it might have improved. However, supervision of HTC services was lacking, with many VHTs reportedly not receiving any support. Earlier findings show that VHTs do not always think of routine interactions with health centers as supportive supervision, even though they acknowledge debriefing on key challenges encountered and turning in reports ${ }^{17}$. More information on the content of interactions between VHTs and health center staff is needed to determine how supervision mechanisms can be strengthened for optimal support within the constraints inherent to the broader health system. Shortages of supplies at the health center were a problem for both FP and HTC, and also warrant attention, perhaps as part of broader discussions on chronic stock outs and new supply chain strategies.

This study expands the evidence base showing that integration of FP and HIV can be considered beneficial and feasible, even at the community level. Our findings are consistent with the evidence base on facility-based FP/HIV 


\section{Brunie et al}

integration models, which shows that linkages between FP and HTC can facilitate HTC uptake $^{3,21}$, and also indicates improvements in contraceptive use through various combinations of FP and HIV services ${ }^{3,4}$. Although somewhat limited, the available literature does not provide evidence of negative effects from potentially increased staff time with clients as a result of integrating services, and effects on the quality of FP counseling when adding HTC are reported as positive or mixed ${ }^{3,21}$. The challenges we observed with the delivery of integrated messages echo process evaluation data from several studies on facility-based integration of FP into HIV services, which showed that provision of contraceptive counseling, while improved, was not systematic and was in some cases lower than expected ${ }^{4}$.

\section{Limitations}

Some limitations must be acknowledged. Though it provides important information on opportunities, challenges, and practical service delivery considerations, this assessment represents a smallscale pilot investigation of an integrated service delivery model for combined, community-based provision of FP and HIV services. Future research is needed to provide more precise findings on this model, particularly at scale. Due to selection criteria and additional training and experience, VHTs who provide injectables might be among the higher performing VHTs. Therefore results might not be applicable to lower-performing VHTs. Moreover, courtesy bias cannot be ruled out in reporting on workload manageability, especially as many VHTs acknowledged increased conflict with their personal lives. Therefore, findings need to be interpreted cautiously in the context of an incremental change in responsibilities with minimal transport implications and a client base that though expanded, was largely similar. While the study compares the experiences of clients in the control and intervention groups, only the perspectives of the VHTs in the intervention group were directly captured. We initially aimed to compare service statistics before and after implementation in the intervention and control groups to assess effectiveness and changes in workloads. However, program events causing
Community-based FP/HTC provision in Uganda

VHTs to leave communities and missing data prevented us from gathering reliable information on pre-intervention service provision. Future research is needed for a realistic assessment of VHT workloads.

\section{Conclusion}

Community-based FP/HTC provision by VHTs appears to be a smart integration model that could be appropriate in other areas with unmet needs, health staff shortages, and limited resources. This intervention from Uganda was delivered through the existing public sector system, leveraging the mechanisms in place to support community-based provision of injectable contraceptives. As such, the model shows potential for adaptation and scale-up within Uganda and other settings. Further research and programmatic evidence are needed to understand its full potential; programs should also consider the specifics of their context to determine the added value of this model in their settings.

\section{Acknowledgments}

Funding was provided by the U.S. Agency for International Development (USAID) under the terms of the Preventive Technologies Agreement No. GHO A 0009 00016-00. The contents do not necessarily reflect the views of USAID or the United States Government. The authors thank Dr. Benson Tumwesigye, National HTC Coordinator; the Uganda Ministry of Health AIDS Control Program; and the District Health Management Teams led by Dr. Godfrey Oundo and Dr. Stephen Sebudde for their collaboration. We also thank Vincent Wong, Senior Technical Advisor with USAID's Office of HIV/AIDS, for his continued support and advice, and Ms. Jane Harriet Namwebya for assistance with implementation.

\section{Contribution of Authors}

$\mathrm{AB}, \mathrm{PM}, \mathrm{AA}$, and TP conceived the study. $\mathrm{AB}$ and $M C$ led the research design. PM oversaw data collection, and SM analyzed data. $\mathrm{AB}$ led the preparation of the manuscript, with inputs from PM, AA, MC, SM, and TP. All authors approved the manuscript. 
Brunie et al

\section{References}

1. WHO. WHO Technical Guidance Note: Strengthening the inclusion of reproductive, maternal, newborn and child $(\mathrm{RMNCH})$ health in concept notes to the Global Fund. Geneva, Switzerland: World Health Organization; 2014.

2. OGAC. PEPFAR blueprint: Creating an AIDS-free generation. Washington, DC: OGAC; 2012.

3. Spaulding AB, Brickley DB, Kennedy C, Almers L, Packel L, Mirjahangir J, Kennedy G, Collins L, Osborne K and Mbizvo M. Linking family planning with HIV/AIDS interventions: a systematic review of the evidence. AIDS 2009;23 Suppl 1:S79-88.

4. Wilcher R, Hoke T, Adamchak SE and Cates Jr W. Integration of family planning into HIV services: a synthesis of recent evidence. AIDS 2013;27:S65S75.

5. Kennedy CE, Spaulding AB, Brickley DB, Almers L, Mirjahangir J, Packel L, Kennedy GE, Mbizvo M, Collins L and Osborne K. Linking sexual and reproductive health and HIV interventions: a systematic review. J Int AIDS Soc 2010;13:26.

6. WHO. Task shifting. Rational redistribution of tasks among health workforce teams: global recommendations and guidelines. Geneva: World Health Organization; 2008.

7. Haines A, Sanders D, Lehmann U, Rowe A, Lawn J, Jan S, Walker D and Bhutta Z. Achieving child survival goals: potential contribution of community health workers. Lancet 2007;369(9579):2121-2131.

8. Singh P and Sachs JD. 1 million community health workers in sub-Saharan Africa by 2015. Lancet 2013;382(9889):363-365.

9. Lehmann U, Van Damme W, Barten F and Sanders D. Task shifting: the answer to the human resources crisis in Africa? Hum Resour Health 2009; 7(1):49.

10. Creanga AA, Bradley HM, Kidanu A, Melkamu Y and Tsui AO. Does the delivery of integrated family planning and HIV/AIDS services influence community-based workers' client loads in Ethiopia? Health policy and planning 2007;22(6):404-414.

11. Adams I and Kraushaar H. Expanded Community Based Distribution Project: Impact Analysis Report, Zimbabwe. Arlington: Advance Africa; 2005.

\section{Community-based FP/HTC provision in Uganda}

12. Uganda Bureau of Statistics, ICF International Inc. Uganda Demographic and Health Survey 2011. Kampala, Uganda: UBOS and Calverton, MD: ICF International Inc.2012.

13. MOH, ICF International. Uganda AIDS Indicator Survey 2011. Kampala, Uganda and Calverton Maryland, USA: Ministry of Health and ICF International; 2012.

14. Staveteig S, Wang S, Head SK, Bradley SEK and Nybro E. Demographic Patterns of HIV Testing Uptake in Sub-Saharan Africa. Calverton, MD: ICF International; 2013 April 2013.

15. WHO, USAID and FHI. Strategic Considerations for Strengthening the Linkages between Family Planning and HIV/AIDS Policies, Programs, and Services Geneva, Switzerland: World Health Organization; 2009.

16. Brunie A, Wamala-Mucheri P, Akol A, Mercer S and M Chen. Expanding HIV testing and counselling into communities: Feasibility, acceptability, and effects of an integrated family planning/HTC service delivery moel by Village Health Teams in Uganda. Health Policy and Planning 2016;31(8).

17. Brunie A, Wamala-Mucheri P, Otterness C, Akol A, Chen M, Bufumbo L and Weaver M. Keeping community health workers in Uganda motivated: key challenges, facilitators, and preferred program inputs. Global Health: Science and Practice 2014;2(1).

18. Alam K, Tasneem S and Oliveras E. Performance of female volunteer community health workers in Dhaka urban slums. Soc Sci Med 2012;75:511-515.

19. Bhattacharyya K, Winch P, LeBan K and Tien M. Community Health Worker Incentives and Disincentives: How they affect motivation, retention and sustainability. Arlington, VA: BASICS/USAID; $2001.52 \mathrm{p}$.

20. Gopalan SS, Mohanty S and Das A. Assessing community health workers' performance motivation: a mixedmethods approach on India's Accredited Social Health Activists (ASHA) programme. BMJ open 2012;2(5).

21. Liambila W, Askew I, Mwangi J, Ayisi R and Kibaru J. Feasibility and effectiveness of integrating providerinitiated testing and counselling within family planning services in Kenya. Aids 2009;23(Suppl 1):S115-S121. 